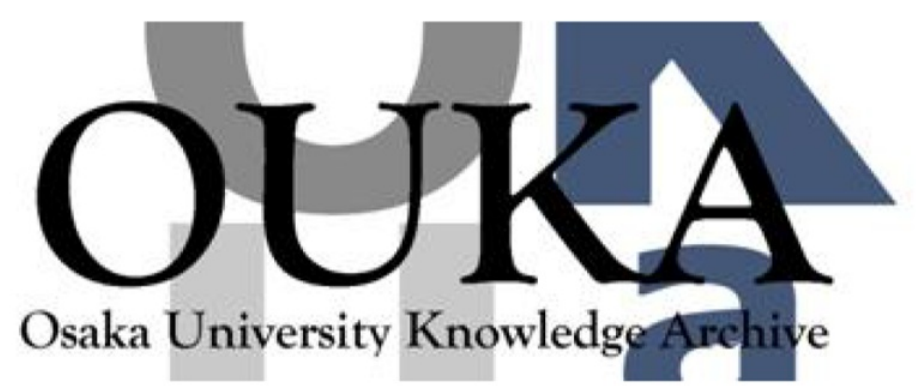

\begin{tabular}{|c|l|}
\hline Title & $\begin{array}{l}\text { Mobile robot map generation by integrating } \\
\text { omnidirectional stereo and laser range finder }\end{array}$ \\
\hline Author(s) & Miura, Jun; Negishi, Yoshiro; Shirai, Yoshiaki \\
\hline Citation & $\begin{array}{l}\text { IEEE International Conference on Intelligent } \\
\text { Robots and Systems. 1 p. 250-p. 255 }\end{array}$ \\
\hline Issue Date & $2002-09$ \\
\hline oaire:version & VoR \\
\hline URL & $\begin{array}{l}\text { https://hdl. handle. net/11094/14082 } \\
\text { c2002 IEEE. Personal use of this material is } \\
\text { permitted. However, permission to } \\
\text { reprint/republish this material for advertising } \\
\text { or promotional purposes or for creat ing new } \\
\text { collective works for resale or redistribution } \\
\text { to servers or lists, or to reuse any } \\
\text { copyrighted component of this work in other } \\
\text { works must be obtained from the IEEE. . }\end{array}$ \\
\hline rights \\
\hline Note & \begin{tabular}{l} 
\\
\hline
\end{tabular} \\
\hline
\end{tabular}

Osaka University Knowledge Archive : OUKA

https://ir. Library. osaka-u. ac. jp/

Osaka University 


\title{
Mobile Robot Map Generation by Integrating Omnidirectional Stereo and Laser Range Finder
}

\author{
Jun Miura, Yoshiro. Negishi, and Yoshiaki Shirai \\ Dept. of Computer-Controlled Mechanical Systems, Osaka University \\ \{jun,negishi,shirai\}@cv.mech.eng.osaka-u.ac.jp
}

\begin{abstract}
This paper describes a map generation method using an omnidirectional stereo and a laser range finger. Omnidirectional stereo has an advantage of $3 D$ range acquisition, while it may suffer from a low reliability and accuracy in range data. Laser range finders have advantage of reliable acquisition of data, while they usually obtain only $2 D$ range information. By integrating these two sensors, a reliable map can be generated. Since the two sensors may detect different parts of an object, a separate probabilistic grid map is first generated by temporal integration of data from each sensor. The resultant two maps are then integrated using a logical integration rule. An ego-motion estimation method is also described, which is necessary for integration of sensor data obtained at different positions. Experimental results on autonomous navigation in unknown environments show the feasibility of the method.
\end{abstract}

\section{Introduction}

Detection of obstacles and free spaces is an essential function of the sensing system for mobile robots. Even if a robot is given a map, this function is indispensable to cope with unknown obstacles or the error of the map.

Many works (e.g., [5, 9]) use stereo vision to detect obstacles. Stereo vision has an advantage of simultaneous acquisition of range data and images; it can find visual features while measuring the distance to obstacles. Conventional stereo systems using a pair of ordinary cameras, however, suffer from a narrow field of view. To cope with this problem, we have developed an omnidirectional stereo system [6]. This system can obtain panoramic range information of almost 360 degrees. At the same time, however, it inevitably inherits the drawbacks of stereo vision: occasional false matches (which will cause problematic false obstacles) and a relatively low accuracy in range, especially for distant objects.

Laser range finders (LRFs) are also widely used (e.g., [7, 11]). Most of them scan a $2 D$ plane and obtain only range data on that plane at a time. They are, therefore, more suitable for a relatively simple environment such as the one surrounded by flat walls. When an LRF observes

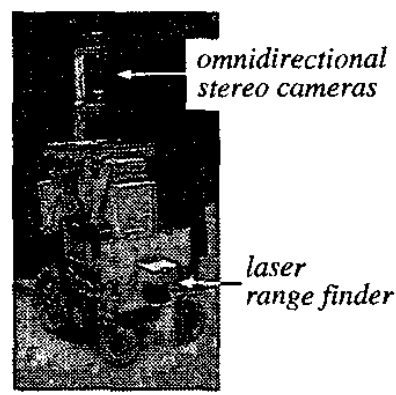

Figure 1: Our mobile robot.

a table, for example, only its legs may be detected, and thus the area under the table may be determined as a free space.

The integration of multiple sensor data is a typical approach to make a reliable map (e.g., $[1,2,4]$ ). This paper thus proposes a method of generating a free space map by integrating an omnidirectional stereo and a laser range finder. Since the two sensors may detect different parts of an obstacle, the method first generates a separate probabilistic grid map for each sensor by temporal integration of data from the sensor. This integration is carried out using a probabilistic model of sensor uncertainty. The resultant two maps are then integrated using a logical integration rule, which is designed by considering the characteristics of sensors and environments. We also describe an ego-motion estimation method, which is necessary for integration of sensor data obtained at different positions. We have implemented the method on a mobile robot (see Fig. 1). The robot can move in unknown environments autonomously.

\section{Two Range Sensors}

\subsection{Real-time Omnidirectional Stereo [6]}

The stereo system uses a pair of vertically-aligned omnidirectional cameras (see Fig. 1). The input images are converted to panoramic images, in which epipolar lines become vertical and in parallel; thus, efficient stereo matching algorithms for the conventional stereo configuration can be applied. In [6], we used a PC cluster to 


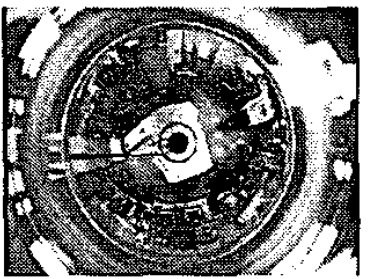

(a) Input omnidirectional image (lower camera)

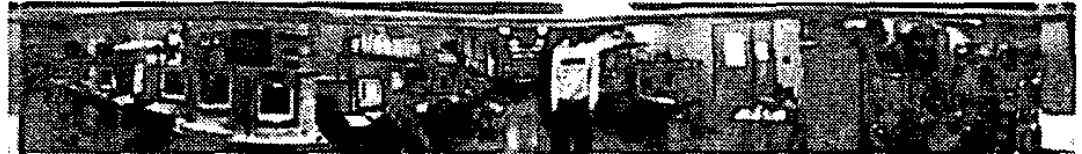

(b) Panoramic image converted from (a).

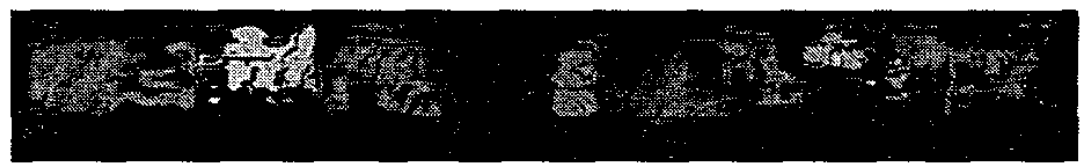

(c) panoramic disparity image obtained from (b).

Figure 2: Omnidirectional stereo generates a panoramic disparity image.

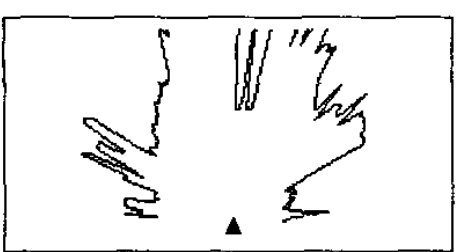

Figure 3: An example LRF measurement. The black triangle indicates the position and the direction of the robot.

realize a real-time range calculation for a relatively large image size (720x 100$)$; the working area of the robot was thus limited due to the wired connection between the robot and the PC cluster. In this paper, we use an onboard PC; to keep the processing speed within the same level, we reduce the size of the input image. The current implementation generates the disparity image of $360 \times 50$ in size and 40 in disparity range. The processing time is about $0.18[s]$ per frame. Fig. 2 shows the panoramic conversion and disparity calculation. In the disparity image (bottom right), larger disparities (nearer points) are drawn in brighter color.

Since the objective of mapping is to recognize the free space, we extract the nearest obstacle in each direction. In the panoramic image, the horizontal axis indicates the direction. So we examine each column of the panoramic disparity image and extract the connected interval in which the disparity difference between (vertically) adjacent pixels is less than or equal to one and which is nearest among such intervals in the same column.

\subsection{Laser Range Finder (LRF)}

We use a SICK laser range finder (LRF), which is set at the front of the robot so that it scans the horizontal plane at the height of $35[\mathrm{~cm}]$ from the floor (see Fig. 1). The resolution used is $1.0[\mathrm{deg}]$ per point (i.e., 181 measurements for 180 degrees). The accuracy of each measurement is $\pm 5[\mathrm{~cm}]$. Fig. 3 shows the line of measurements corresponding to the scene shown in Fig. 2.

\section{Map Generation by Integrating Two Sen- sors}

\subsection{Strategy for integrating two sensors}

One strategy for integrating range data (with uncertainty) from the two sensors is to treat both data similarly except the uncertainty model to be used. In this case, whenever an observation result is obtained by one of the sensors, the result is integrated into a probabilistic (or certainty) map. This unified approach is appropriate for the case where the sensors obtain basically the same information but with different accuracies and reliabilities.

When the sensors occasionally recognizes the same object differently, however, the above strategy may lead to a problematic situation. A simple but typical example is as follows. Suppose the robot observes a table ahead; the stereo observes the table top and the (visible) legs, while the LRF observes only the legs. Let us focus on a position under the table. Since the stereo insists that an obstacle exists at the position while the LRF does not, the integration result for the position will be something between an obstacle and a free space; that is, the probability of an obstacle existing there will be around, say, 0.5 . This resuit is apparently erroneous; or, if the data from the LRF are more reliable than those from the stereo, the position may be determined as a free space. In this example, the stereo strongly insists the existence of an obstacle; this fact should be treated importantly, even if the LRF also strongly insists that the position is free.

To realize such sensor integration, we take the following strategy. We first treat the data from the sensors separately, i.e., generate a separate probabilistic map for each sensor by temporal integration of data from the sensor. We then determine the type of every position in each map, and finally integrate the type information in the two maps into the final free space map. The final integration step is done by a logical integration rule, which is devised by considering the characteristics of sensors and environments. 


\subsection{Temporal integration of sensor data}

Certainty grids proposed by Elfes [4] are often used for temporal integration of sensor data with uncertainty. Each grid has the probability (or certainty) of an obstacle being there and the statistical integration procedure is used to update the probability after each observation. The update of a grid is usually carried out independently of other grids (independence assumption).

Thrun [13] proposed to use a forward sensor model, as opposed to an inverse model in [4], to overcome the independence assumption in mapping using ultrasonic sensors. A forward model describes the physics of the environment, from causes (occupancy) to effects (measurements) and more natural than inverse models. We have also proposed to use a forward model in stereo-based environment recognition [12] but made the independence assumption.

Probabilistic map learning using forward models without the independence assumption requires a search in a high-dimensional space (e.g., the EM algorithm in [13]), which is usually computationally expensive. So we adopt forward sensor models under the independence assumption, which seems reasonable when the range sensor has a fairly fine angular resolution as in the case of our omnidirectional stereo and the LRF.

\subsection{Interpretation of range data and integration formula}

From one observation, we determine the attribute of each grid; possible attributes are: occupied, free, and unknown (see Fig. 4). The figure shows the attribute determination for a region within one angular resolution. $R$ is the observed distance (by omnidirectional stereo or LRF) to the nearest obstacle, and $R_{\min }$ and $R_{\max }$ indicate the uncertainty in range measurement ${ }^{1}$. The region between $R_{\min }$ and $R_{\text {max }}$ is labeled as occupied. The region before the occupied region is labeled as free. The region behind the occupied region is labeled as unknown. In the case of stereo, all regions corresponding to the directions in which any obstacles are not detected (possibly due to the failure of stereo matching) are labeled as unknown.

Let $O$ be the event that an obstacle is detected. $O$ occurs at occupied grids; the inverse event $\bar{O}$ occurs at free grids. For such grids, the update of the probability is carried out. For unknown grids, no update is carried out because no information on obstacles is obtained this time. The update procedure is as follows.

Let $E$ be the event that an obstacle exist, and let $P(E)$ be the probability that an obstacle exist (at a grid). The new probability map to be obtained by integrating a new observation is given by the conditional probabilities:

\footnotetext{
${ }^{1}$ Refer to [6] for the uncertainty estimate of omnidirectional stereo. The uncertainty in LRF measurement is constant regardless of the measured value.
}

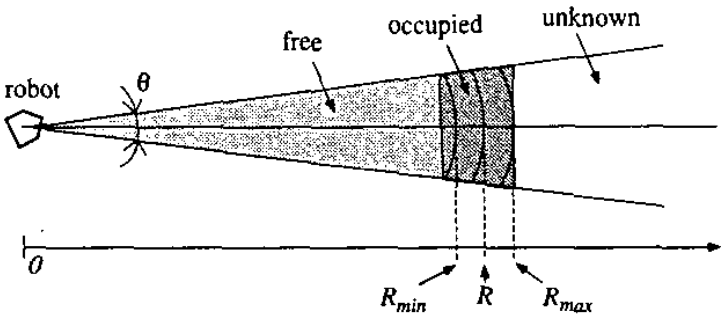

Figure 4: Determination of grid attributes.

$P(E \mid O)$ and $P(E \mid \bar{O})$. These probabilities are calculated by the Bayes' theorem as follows:

$$
\begin{aligned}
P(E \mid O) & =\frac{P(O \mid E) P(E)}{P(O \mid E) P(E)+P(O \mid \bar{E}) P(\bar{E})}, \\
P(E \mid \bar{O}) & =\frac{P(\bar{O} \mid E) P(E)}{P(\bar{O} \mid E) P(E)+P(\bar{O} \mid \bar{E}) P(\bar{E})},
\end{aligned}
$$

where $P(E)$ is the prior probability and $\bar{E}$ is the proposition that an obstacle does not exist. Among the terms in the above equations, $P(O \mid E)$ and $P(O \mid \bar{E})$ are observation models described below; $P(\bar{O} \mid E)=1-P(O \mid E)$; $P(\bar{O} \mid \bar{E})=1-P(O \mid \bar{E}) ; P(\bar{E})=1-P(E)$. Integration for each grid is performed independently of the others (the independence assumption).

\subsection{Probabilistic models of sensor uncertainty}

Two probabilistic models of observation, $P(O \mid E)$ and $P(O \mid \bar{E})$, are determined by considering the reliability of the sensors as follows.

\subsubsection{Stereo uncertainty model}

$P(O \mid E)$ is the probability that an obstacle is observed when it actually exists. In the case of stereo, there is always the possibility that the correct match is not obtained depending on, for example, the textures on obstacle surfaces and the lighting condition; this possibility usually rises as an obstacle becomes distant and its size in the image decreases. Since the size is inversely proportional to the distance, we assume that $P(O \mid E)$ is also inversely proportional to the distance. The minimum measurable distance of an object on the floor is $1.3[\mathrm{~m}]$ by our omnidirectional stereo. So the probability at the distance is set to 0.8 . For each grid which is nearer than that distance, the probability update is carried out only when an obstacle is detected there (i.e., labeled as occupied); since this detected obstacle may not actually be the nearest obstacle due to the limitation of field of view, $P(O \mid E)$ is set to 0.5 within $1.3[\mathrm{~m}]$. Fig. 5 shows the definition of $P(O \mid E)$ for stereo. $P(O \mid \bar{E})$ corresponds to the case where a false object is detected due to a false stereo matching, and is set to 0.05 . 


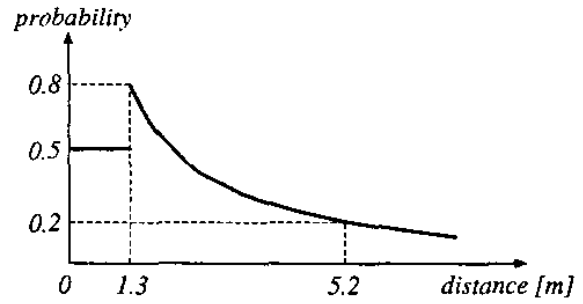

Figure 5: Stereo uncertainty model, $P(O \mid E)$.

Table 1: The integration rule.

\begin{tabular}{|c|c|c|c|c|}
\hline \multicolumn{2}{|c|}{} & \multicolumn{3}{|c|}{ stereo } \\
\cline { 2 - 5 } \multicolumn{2}{c|}{} & obstacle & undecided & free space \\
\hline $\mathrm{L}$ & obstacle & obstacle & obstacle & obstacle \\
\cline { 2 - 5 } $\mathrm{R}$ & undecided & obstacle & obstacle & free space \\
\cline { 2 - 5 } $\mathrm{F}$ & free space & obstacle & free space & free space \\
\hline
\end{tabular}

\subsubsection{LRF uncertainty model}

The measurement of the LRF is fairly reliable and the reliability does not depend on the distance to obstacles. Therefore we set $P(O \mid E)$ to 0.9 and $P(O \mid \bar{E})$ to 0.05 .

\subsection{Integration of two maps}

The two probabilistic grid maps are integrated as follows. A direct integration of probability values is not appropriate because, as explained before, a high existence probability of an obstacle strongly supported by a sensor may be unnecessarily lowered by the data from the other sensor. We therefore first classify each grid of a map into one of the following three types, obstacle, free space, and undecided, using two thresholds. If the probability of a grid is larger than the higher threshold (currently, 0.7), the grid is classified as obstacle; if the probability is less than the lower threshold (currently, 0.3), the grid is classified as free space; otherwise, classified as undecided. These thresholds have been empirically selected.

After the classification, we finally integrate the two maps into the free space map using the rule shown in Table 1. This rule basically says the following:

- Classification obstacle is reliable and believable. In other words, it is assumed that any obstacle is detectable by at least one of the sensors.

- If none of the sensors is confident with the detection of an obstacle or a free space for a grid, the grid is temporarily considered as an obstacle. This is for a safe navigation.

The classification and integration processes are carried out every frame, after updating both probabilistic maps. The resultant free space map is used for the path planning of the mobile robot.

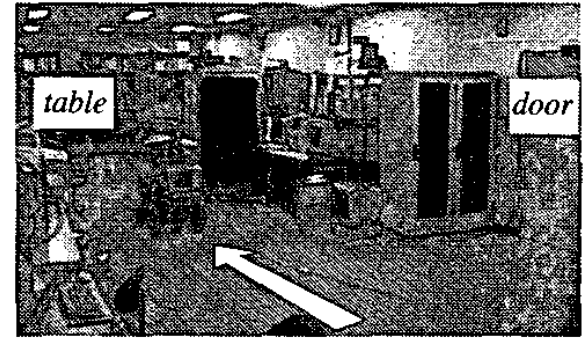

Figure 6: An example scene.

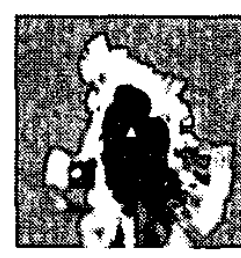

stereo probabilistic map

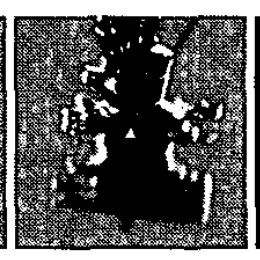

$L R F$ probabilistic map

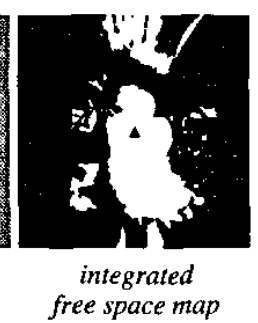

free space map
Figure 7: Probabilistic maps and a frée space map. Black and white triangles indicate the robot position and orientation.

This integration process is similar to fuzzy logic-based ones (e.g., [10]), although each source of information (i.e., two probabilistic grid maps) is obtained based on the probability theory in our case.

\subsection{Map generation example}

Fig. 6 shows an example scene where the robot moved along the arrow. Fig. 7 shows the maps generated after the movement. In the probabilistic maps, brightness indicates the probability. The maps are drawn in the robot coordinates. The table in front of the robot was correctly recognized by the stereo, while the LRF only detected its legs. On the other hand, the recognition by the stereo of the region near the door on the right failed at many positions because features are scarce on the door, while the LRF correctly recognized the region. In spite of recognition failures by one of the sensors at several positions, the integrated map reasonably represents the free space.

\section{Ego-motion estimation using LRF data}

To integrate multiple observations obtained at different robot positions, ego-motion of the robot is necessary. Data from internal sensors such as an odometry-based dead reckoning may be used, but the error in such data is accumulated as the robot moves. Ego-motion estimation using external sensors (vision or LRF) is, therefore, necessary. Among the two sensors we use, the LRF is better for ego-motion estimation thanks to its reliability and accuracy, as long as an enough number of features are obtained. 


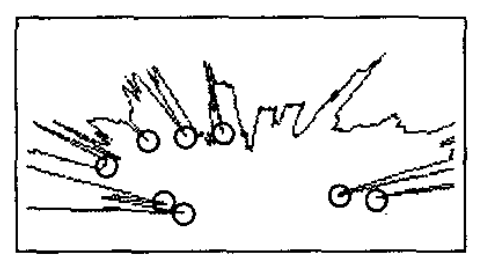

Figure 8: Extracted feature points in LRF data.

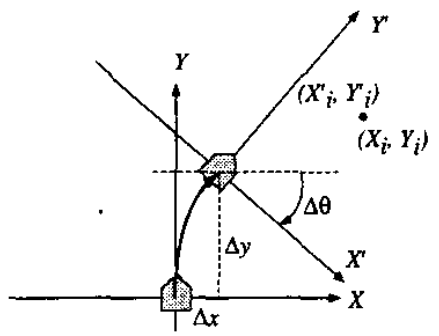

Figure 9: Ego-motion estimation using point features.

\subsection{Feature extraction and matching}

Line segments, which come from flat walls, are often used as features for range sensor-based ego-motion estimation [3]. Lu and Milios [8] proposed a localization method which allows arbitrarily-shaped walls; but the method seems to be applicable only to relatively smooth surfaces. In indoor environments, there are plenty of protruded parts in LRF data, which correspond to legs of tables and chairs. So we use such features for localization.

The width of the legs in our rooms are mostly 5 to $6[\mathrm{~cm}]$; so we extract, as protruded parts, ranges in the line of LRF measurements which are nearer than the data on the both sides by more than $40[\mathrm{~cm}]$, and which have the width less than $10[\mathrm{~cm}]$. To avoid false correspondence, we currently do not use a feature if it is within $30[\mathrm{~cm}]$ distant from another. Fig. 8 shows an example line of LRF measurements and extracted features.

Extracted features in two consecutive frames are matched based on the distance between features; if the distance between two features is less than a certain threshold (currently, $20[\mathrm{~cm}]$ ), they are considered to match and used for the ego-motion estimation described below.

\subsection{Ego-motion estimation}

The ego-motion $(\Delta x, \Delta y, \Delta \theta)$ between two consecutive frames is estimated as follows. Let $\left(X_{i}, Y_{i}\right)$ and $\left(X_{i}^{\prime}, Y_{i}^{\prime}\right)$ be the $i$ th matched pair of features $(i=1, \ldots, N)$. If there is no error, the following equation must hold (see Fig. 9):

$$
\left(\begin{array}{cc}
\cos \Delta \theta & \sin \Delta \theta \\
-\sin \Delta \theta & \cos \Delta \theta
\end{array}\right)\left(\begin{array}{c}
X_{i}^{\prime} \\
Y_{i}^{\prime}
\end{array}\right)+\left(\begin{array}{c}
\Delta x \\
\Delta y
\end{array}\right)=\left(\begin{array}{c}
X_{i} \\
Y_{i}
\end{array}\right)
$$

So we calculate the best ego-motion which minimizes the following sum $S$ of the squared distances:

$$
\begin{aligned}
S= & \sum_{i=1}^{N}\left\{\left[X_{i}-\left(X_{i}^{\prime} \cos \Delta \theta+Y_{i}^{\prime} \sin \Delta \theta+\Delta x\right)\right]^{2}\right. \\
& \left.+\left[Y_{i}-\left(-X_{i}^{\prime} \sin \Delta \theta+Y_{i}^{\prime} \cos \Delta \theta+\Delta y\right)\right]^{2}\right\}
\end{aligned}
$$

The ego-motion which minimizes $S$ is given by solving the following equations:

$$
\frac{\partial S}{\partial \Delta x}=0, \quad \frac{\partial S}{\partial \Delta y}=0, \quad \frac{\partial S}{\partial \Delta \theta}=0 .
$$

From eqs. (3) and (4), we obtain the following analytical solution:

$$
\begin{aligned}
& \Delta \theta=\tan ^{-1} \frac{N\left[X_{i} Y_{i}^{\prime}\right]-N\left[X_{i}^{\prime} Y_{i}\right]-\left[X_{i}\right]\left[Y_{i}^{\prime}\right]+\left[Y_{i}\right]\left[X_{i}^{\prime}\right]}{N\left[X_{i} X_{i}^{\prime}\right]+N\left[Y_{i} Y_{i}^{\prime}\right]-\left[X_{i}\right]\left[X_{i}^{\prime}\right]-\left[Y_{i}\right]\left[Y_{i}^{\prime}\right]}, \\
& \Delta x=\frac{\left[X_{i}\right]-\left[X_{i}^{\prime}\right] \cos \Delta \theta-\left[Y_{i}^{\prime}\right] \sin \Delta \theta}{N}, \\
& \Delta y=\frac{\left[Y_{i}\right]+\left[X_{i}^{\prime}\right] \sin \Delta \theta-\left[Y_{i}^{\prime}\right] \cos \Delta \theta}{N},
\end{aligned}
$$

where $[\cdot]$ indicates the summation from 1 to $N$.

\section{Navigation Experiment}

The proposed method has been implemented on our mobile robot shown in Fig. 1. The total processing time of omnidirectional stereo, LRF data acquisition, feature extraction and localization using LRF data, and the update of the probabilistic maps and the free space map is currently about $0.55[s]$ per frame. Although the time of data acquisition is slightly different for two kinds of sensors, this difference is not significant for the current, relatively slow speed of the robot.

Fig. 10 shows the result of an autonomous navigation. At the initial position, the robot assumed to be completely in a free space. Every time the robot obtained a new pair of observations by the two sensors, it updated the two probabilistic maps and the free space map, and planned the safe movement to follow. The robot successfully moved around a complex indoor environment.

\section{Conclusion}

We have described a free space map generation method using an omnidirectional stereo and a laser range finder. Since the two sensors may detect different parts of an object, instead of integrating two kinds of sensor data into one probabilistic map, the method maintains a separate probabilistic map for each sensor and integrates them into a free space map using a logical integration rule. Using the generated map, the robot was successfully navigated in unknown environments. 
(a)

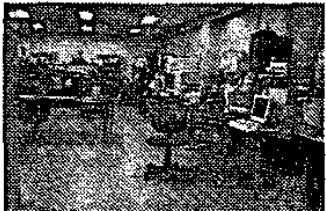

(b)

(c)
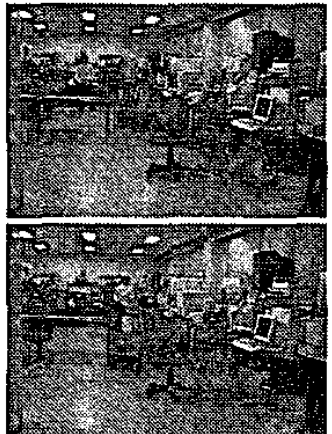

(d)

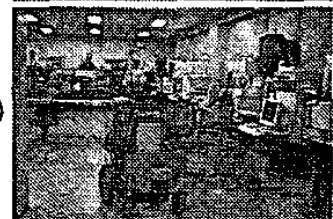

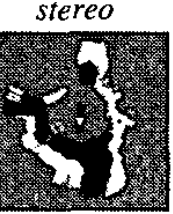
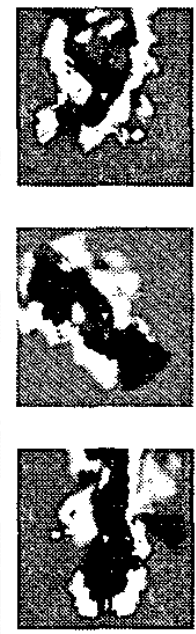
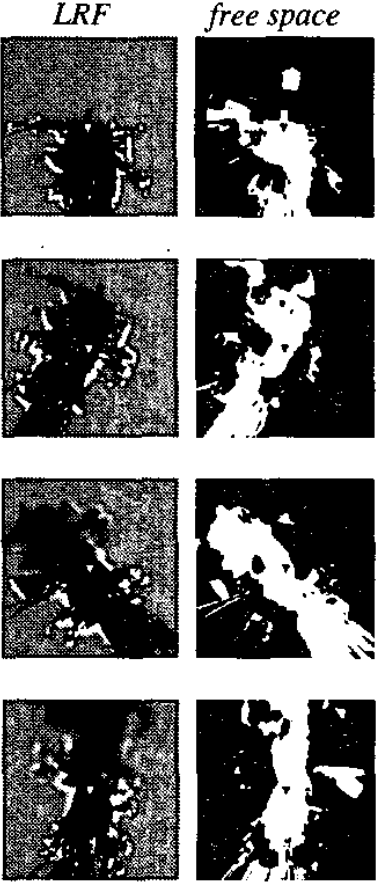

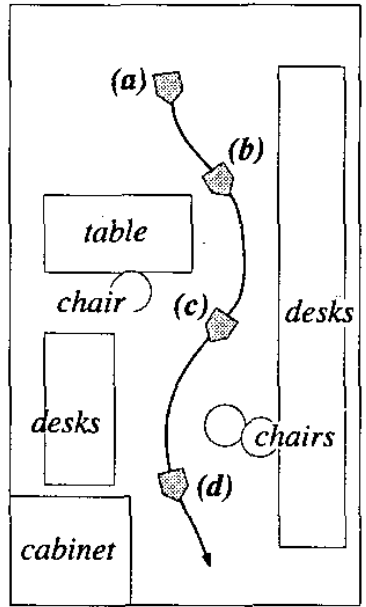

Figure 10: A navigation result. Note that in the maps, the direction of the robot is now right downward, which is different from the maps in Fig, 7, so that the maps match well with the photos on the left. Black and white triangles indicate the robot position and orientation.

Currently, the proposed method assumes static environments. A future work is to extend the method to cope with dynamic obstacles by, for example, adopting obstacle detection method such as [6].

\section{Acknowledgments}

This research is supported in part by the Kurata Foundation, Tokyo, Japan. The authors would like to thank Hiroshi Koyasu for the implementation of the omnidirectional stereo system.

\section{References}

[1] M. Asada and Y. Shirai. Building a World Model for a Mobile Robot Using Dynamic Semantic Constraints. In Proc. of the 11th Int. Joint Conf. on Artificial Intelligence, pp. 1629-1634, 1989.

[2] N. Ayache and O.D. Faugeras. Maintaining Representations of the Environment of a Mobile Robot. IEEE Trans. on Robotics and Automat., Vol. RA-5, No. 6, pp. 804-819, 1989.

[3] I.J. Cox. Blanche: Position Estimation for an Autonomous Robot Vehicle. In Proc. of IEEE/RSJ Int. Workshop on Intelligent Robots and Systems, pp. 432-439, 1989.

[4] A. Elfes. Sonar-Based Real-World Mapping and Navigation. Int. J. of Robotics and Automat., Vol. 3, No. 3, pp. 249-265, 1987 .

[5] S. Kagami, K. Okada, M. Inaba, and H. Inoue. Design and Implementation of Onbody Real-time Depthmap Generation System. In Proc. of 2000 IEEE Int. Conf. on Robotics and Automation, pp. 1441-1446, 2000.
[6] H. Koyasu, J. Miura, and Y. Shirai. Realtime Omnidirectional Stereo for Obstacle Detection and Tracking in Dynamic Environments. In Proc. of the 2001 IEEE/RSJ Int. Conf. on Intelligent Robots and Sysetms, pp. 31-36, 2001.

[7] M. Lindström and J.-O. Eklundh. Detecting and Tracking Moving Objects from a Mobile Platform using a Laser Range Scanner. In Proc. of IEEE Int. Conf. on Intelligent Robots and Systems, pp. 1364-1369, 2001.

[8] F. Lu and E.E. Milios. Robot Pose Estimation in Unknown Environments by Matching 2D Range Scans. In Proc. of IEEE Conf. on Computer Vision and Pattern Recognition, 1994.

[9] D. Murray and J. Little. Using Real-Time Stereo Vision for Mobile Robot Navigation. Autonomous Robots, Vol. 8, No. 2, pp. 161-171, 2000.

[10] G. Oriolo, G. Ulivi, and M. Vendittelli. Real-Time Map Building and Navigation for Autonomous Robots in Unknown Environments. IEEE Trans. on System, Man, and Cybernetics - Part B: Cybernetics, Vol. 28, No. 3, pp. 316-333, 1998.

[11] E. Prassler and J. Scholz. Tracking Multiple Moving Objects for Real-Time Navigation. Autonomous Robots, Vol. 8, No. 2, pp. 105-116, 2000.

[12] Y. Sawano, J. Miura, and Y. Shirai. Man Chasing Robot by an Environment Recognition Using Stereo Vision. In Proc. of the 2000 Int. Conf. on Machine Automation, pp. 389-394, 2000.

[13] S. Thrun. Learning Occupancy Grids with Forward Models. In Proc. of 2001 IEEE/RSJ Int. Conf. on Intelligent Robots and Systems, pp. 1676-1681, 2001. 